\title{
A comparison between octreotide-LAR and lanreotide-SR in the chronic treatment of acromegaly
}

\author{
R Cozzi, D Dallabonzana, R Attanasio, M Barausse and G Oppizzi \\ Division of Endocrinology, Niguarda Hospital, Milan, Italy \\ (Correspondence should be addressed to R Cozzi, Division of Endocrinology, Ospedale Niguarda, Piazza Ospedale Maggiore 3, I-20162 Milano, Italy; \\ Email: endonig@rdn.it)
}

\begin{abstract}
Background: At present long-acting somatostatin analogs represent the first-line medical treatment of acromegaly. These drugs produce stable suppression of GH in most sensitive patients and IGF-I normalization in many; they also increase the compliance of acromegalic patients. The recent availability of octreotide (OC)-LAR, a somatostatin analog to be administered at 28-day intervals, has prompted us to compare, in the same group of patients, its effects and those of another somatostatin analog already available, lanreotide-SR (LSR, to be administered at 14-day intervals). Patients: Twelve somatostatin analog-sensitive acromegalic patients with active disease were enrolled in a prospective open sequential study after giving their informed consent. After chronic treatment with LSR (6-24 months), the patients were changed to treatment with OC-LAR, without wash-out. LSR had been administered at individually tailored dosages $(30 \mathrm{mg}$ i.m. at 7-21-day intervals, median 10 days - every 7 days in seven patients, 10 days in two patients, 14 days in two patients and 21 days in one patient) according to GH and IGF-I responses. Disease stability was obtained, as shown by maximal GH/IGF-I suppression without any significant hormonal change between the last two control measurements. OC-LAR was administered i.m. at 28-day intervals six times at the dosage of $20 \mathrm{mg}$ for the first three times and 10 or $30 \mathrm{mg}$ for the last three times (according to individual GH/IGF-I responses). GH (mean of three, hourly samples) and IGF-I concentrations were evaluated on the same day as each administration of the drug, before its injection.

Results: GH and IGF-I values were significantly decreased by LSR treatment. GH decreased from $41.6 \pm 14.6 \mu \mathrm{g} / \mathrm{l}$ (mean \pm S.E.) to $7.2 \pm 1.5 \mu \mathrm{g} / \mathrm{l}(P<0.02)$, whereas IGF-I values declined from $959 \pm 95 \mu \mathrm{g} / \mathrm{l}$ to $460 \pm 61 \mu \mathrm{g} / \mathrm{l}(P<0.00001)$, expressed as absolute values, and from $287 \pm 30 \%$ to $137 \pm 19 \%$ expressed as percentage of the upper limit of normal range (\%ULNR). At the end of the last cycle, OC-LAR treatment achieved a significant further suppression both in GH (to $5.1 \pm 1.1 \mu \mathrm{g} / \mathrm{l}$, $P<0.05$ compared with LSR) and in IGF-I concentrations (to $374 \pm 60 \mu \mathrm{g} / \mathrm{l}, P<0.05$ compared with LSR, and to $112 \pm 19 \%$ as \%ULNR). LSR decreased GH concentrations to less than $2.5 \mu \mathrm{g} / \mathrm{l}$ in one patient and normalized IGF-I concentrations in four patients. OC-LAR decreased GH concentrations to less than $2.5 \mu \mathrm{g} / \mathrm{l}$ in four patients and normalized or near-normalized IGF-I concentrations (i.e. to $<110 \%$ ULNR) in eight patients.

Conclusions: These preliminary results show that the once-monthly OC-LAR administration schedule proved more efficacious than LSR given every 7-21 days, in a greater number of acromegalic patients.
\end{abstract}

European Journal of Endocrinology 141 267-271

\section{Introduction}

Long-acting somatostatin analogs at present represent the first-line medical treatment in acromegaly (1). They achieve stable suppression of growth hormone $(\mathrm{GH})$ in most sensitive patients and normalization of insulin-like growth factor (IGF)-I in many; their administration schedule also increases the compliance of acromegalic patients. Although initial trans-sphenoidal surgery still appears to be the best option for many patients with $\mathrm{GH}$-secreting adenomas, somatostatin analogs are now undergoing evaluation as primary treatment in some selected patients when the possibility of surgical cure is low. Their use as primary treatment is supported by a recent study that showed no significant difference in the percentage of responders and in $\mathrm{GH}$ concentrations between patients previously treated by non-curative neurosurgery and then treated by octreotide, and patients whose primary treatment was with octreotide (2).

Among somatostatin analogs, lanreotide-SR (LSR) is widely available and is administered at intervals ranging from 10 to 14 days (3). The recent availability of octreotide-LAR (OC-LAR), a somatostatin analog to be 
Table 1 Demographic and clinical data.

\begin{tabular}{lccccccr}
\hline $\begin{array}{l}\text { Patient } \\
\text { no. }\end{array}$ & Sex & $\begin{array}{c}\text { Age } \\
\text { (yr) }\end{array}$ & Tx & Rx & CT/MRI $\dagger$ & $\begin{array}{c}\text { Basal GH } \\
(\mu \mathrm{g} / \mathrm{l})\end{array}$ & $\begin{array}{c}\text { Basal IGF-I } \\
(\mu \mathrm{g} / \mathrm{l})\end{array}$ \\
\hline 1 & $\mathrm{M}$ & 57 & - & - & $\mu$ & 14 & 879 \\
2 & $\mathrm{M}$ & 47 & - & - & $\mathrm{N}$ & 16.4 & 1086 \\
3 & $\mathrm{M}$ & 49 & + & - & $\mathrm{R}$ & 46 & 1216 \\
4 & $\mathrm{M}$ & 28 & - & - & $\mathrm{M}$ & 130 & 1400 \\
5 & $\mathrm{~F}$ & 58 & + & + & $\mathrm{ES}$ & 9 & 560 \\
6 & $\mathrm{~F}$ & 59 & - & - & $\mathrm{N}$ & 37 & 783 \\
7 & $\mathrm{~F}$ & 60 & + & + & $\mathrm{R}$ & 23 & 750 \\
8 & $\mathrm{~F}$ & 71 & - & - & $\mu$ & 160 & 1634 \\
9 & $\mathrm{~F}$ & 60 & - & - & $\mathrm{M}$ & 40 & 900 \\
10 & $\mathrm{M}$ & 76 & - & - & $\mathrm{M}$ & 7.7 & 705 \\
11 & $\mathrm{~F}$ & 30 & + & - & $\mathrm{R}$ & 7.7 & 1000 \\
12 & $\mathrm{M}$ & 49 & + & - & $\mathrm{R}$ & & \\
\hline
\end{tabular}

$\mathrm{Tx}$, previous neurosurgical treatment; Rx, previous radiotherapy; †neuroradiological imaging: $\mu=$ microadenoma, $\mathrm{M}=$ intrasellar macroadenoma, $\mathrm{R}=$ remnant of adenoma, $\mathrm{ES}=$ empty sella, $\mathrm{N}=$ normal.

administered at 28-day intervals $(4,5)$, has prompted us to compare the effects of these two long-acting somatostatin analogs on hormonal concentrations, in the same group of acromegalic patients.

\section{Patients and methods}

\section{Patients}

Twelve acromegalic patients (six women and six men, aged $28-76$ years, median 58 years) with active disease (according to their clinical profile: high GH concentrations not suppressible by oral glucose load to less than $1 \mu \mathrm{g} / \mathrm{l}$, and increased age-matched IGF-I concentrations) were enrolled in a prospective open sequential study. Five had been previously treated by neurosurgery and two had been irradiated 5-20 years before the study began. At neuroradiological pituitary imaging, three had macroadenoma, two had microadenoma invading the cavernous sinus, four had remnants of pituitary adenoma invading the cavernous sinus, one had empty sella with no evidence of adenoma on the floor of sella turcica, and two had normal pituitary imaging. No patient was hyperprolactinemic and three (Nos 1, 9 and 10) had glucose intolerance. Individual demographic and clinical data are reported in Table 1.

Any drug treatment aimed at decreasing $\mathrm{GH}$ hypersecretion (except those of the study procedure) or potentially capable of interfering with GH secretion was withdrawn at least 8 weeks before the start of the study, as part of periodic off-treatment evaluation of the disease, whereas substitutive treatment with L-thyroxine and cortisone acetate was regularly carried out in patient No. 7 .

Each patient gave informed consent after full explanation of the purpose of the study, which was approved by the ethics committee of our hospital, and the procedures followed were in accordance with the Helsinki Declaration of 1975 as revised in 1983.

\section{Procedure}

Only somatostatin analog-sensitive patients were enrolled - that is, those showing a reduction in $\mathrm{GH}$ greater than $50 \%$ of the basal value after the acute s.c. administration of $100 \mu \mathrm{g}$ octreotide.

Chronic treatment with LSR (Ipsen-Beaufour, Milan, Italy) was carried out with an individually tailored schedule for 6-24 months (Table 2). With the aim of obtaining the maximal GH/IGF-I suppression, the starting schedule of $30 \mathrm{mg}$ i.m. every 14 days was adjusted at 2-month-intervals according to GH/IGF-I values, by shortening the interval between drug administrations to 10 days and, later on, to 7 days, or increasing it to 21 days when GH/IGF-I remained pathological or became less than $50 \%$ of the upper limit of normal range (\%ULNR) respectively.

Table 2 Long-acting treatment with somatostatin analogs.

\begin{tabular}{|c|c|c|c|}
\hline \multirow{2}{*}{$\begin{array}{l}\text { Patient } \\
\text { no. }\end{array}$} & \multicolumn{2}{|c|}{ LSR } & \multirow{2}{*}{$\begin{array}{l}\text { OC-LAR } \\
\text { Dosage§ }\end{array}$} \\
\hline & Duration† & Schedule‡ & \\
\hline 1 & 12 & 7 & 30 \\
\hline 2 & 12 & 7 & 30 \\
\hline 3 & 6 & 7 & 30 \\
\hline 4 & 12 & 7 & 30 \\
\hline 5 & 15 & 14 & 10 \\
\hline 6 & 12 & 10 & 20 \\
\hline 7 & 10 & 7 & 30 \\
\hline 8 & 24 & 21 & 10 \\
\hline 9 & 12 & 7 & 30 \\
\hline 10 & 12 & 14 & 10 \\
\hline 11 & 12 & 7 & 30 \\
\hline 12 & 10 & 10 & 20 \\
\hline
\end{tabular}

† Months of treatment; ‡days between injections; §OC-LAR dosage in the last three cycles of the study period. 
After treatment with LSR, the patients were changed directly to treatment with OC-LAR (kindly supplied by Novartis, Milan, Italy), without wash-out. OC-LAR was administered i.m. at 28-day intervals on six occasions, at dosages of $20 \mathrm{mg}$ for the first three times and 10 or $30 \mathrm{mg}$ for the last three times, increasing or decreasing the dosage according to the individual IGF-I response, as described above.

Control measurements were performed on an outpatient basis, at 56-60-day intervals during LSR and, with OC-LAR, at 28-day intervals during the first three cycles and then at the end of the 6th cycle. Every control was performed on the same day as the administration of the drug, before the injection was given, with a careful clinical evaluation and blood analysis.

Blood samples were collected in the morning hourly for 3 hours, after an overnight fast and rest, while the patients were supine and awake, with an indwelling needle inserted in an antecubital vein and kept patent by slow infusion of saline. $\mathrm{GH}$ concentrations were assayed on each sample (in the results section the reported value is the mean of the three samples) and IGF-I, glucose and glycated hemoglobin $\left(\mathrm{HbA}_{1 \mathrm{c}}\right)$ on the first sample. Ultrasound examination of the gallbladder was performed at 6-month-intervals.

\section{Methods}

Glucose and $\mathrm{HbA}_{1 \mathrm{c}}$ were assayed with standard methods (glucose oxidase and HPLC respectively).

GH (Sorin, Saluggia, Italy) and IGF-I (Nichols, San Juan de Capistrano, California, USA) were assayed in duplicate by DELFIA and RIA after acid-ethanol extraction, respectively. The limit of detection of GH was $0.1 \mu \mathrm{g} / \mathrm{l}$. Standards were calibrated against $1 \mathrm{st}$ IS $80 / 505$ ( $1 \mathrm{ng}=2 \mathrm{IU}$ ) for $\mathrm{GH}$, and WHO 87/518 for IGFI. Intra- and interassay coefficients of variation were 3.5 and $5.5 \%$ for GH, 3.7 and $7.2 \%$ for IGF-I. Normal values for IGF-I in our laboratory are 114-492 $\mu \mathrm{g} / \mathrm{l}$ in patients 25-39 years old, $90-360 \mu \mathrm{g} / \mathrm{l}$ in patients $40-54$ years old and $71-290 \mu \mathrm{g} / \mathrm{l}$ in patients older than 55 years.

\section{Statistical analyses}

Values are expressed as means \pm S.E. IGF-I concentrations are expressed both as absolute values and as \%ULNR (age-adjusted).

Data were analyzed by $t$-test, repeated measures analysis of variance followed by Student-NewmanKeuls test, Spearman correlation test, or Fisher exact test, as appropriate.

Values of $P$ less than 0.05 were considered significant.

\section{Results}

\section{LSR treatment}

$\mathrm{GH}$ decreased from $41.6 \pm 14.6 \mu \mathrm{g} / \mathrm{l}$ to $7.2 \pm 1.5 \mu \mathrm{g} / \mathrm{l}$ $(P=0.018)$ at the end of treatment, whereas IGF-I values declined from $959 \pm 94 \mu \mathrm{g} / \mathrm{l}$ to $460 \pm 61 \mu \mathrm{g} / \mathrm{l}$ $(P<0.00001)$, expressed as absolute values, and from $287 \pm 30 \%$ to $137 \pm 19 \%$ expressed as \%ULNR. LSR obtained a stable control of the disease - that is, no significant changes in GH/IGF-I concentration were observed between the last two control measurements. The neuroradiological control performed at the 12th month showed a more than 30\% shrinkage of the adenoma compared with its size at the baseline imaging, in three patients (Nos 4, 9 and 10) whose primary treatment was with somatostatin analogs.

\section{OC-LAR treatment}

After the first three cycles, GH values were $5.4 \pm 1.2 \mu \mathrm{g} / \mathrm{l}$ $(P=0.05$ compared with data from LSR) and IGF-I $469 \pm 70 \mu \mathrm{g} / \mathrm{l}$ (not different from data on LSR) $(140 \pm 23 \%$ as \%ULNR).

At the end of the 6th cycle, no further decline was observed in GH values $(5.1 \pm 1.1 \mu \mathrm{g} / \mathrm{l})$, whereas IGF-I values declined to $374 \pm 60 \mu \mathrm{g} / \mathrm{l}(P=0.05$ compared with LSR and compared with data after the 3rd cycle on OC-LAR) (112 $\pm 19 \%$ expressed as \%ULNR).

\section{Dosages (Table 2)}

LSR was administered every 7 days in seven patients (Nos 1-4, 7, 9 and 11), every 10 days in two patients (Nos 6 and 12), every 14 days in two patients (Nos 5 and 10), and every 21 days in one patient (No. 8).

In the last three cycles of the study, OC-LAR dosages were decreased to $10 \mathrm{mg} /$ cycle in three patients (Nos 5 , 8 and 10), and increased up to $30 \mathrm{mg}$ in seven patients (Nos 1-4, 7, 9 and 11).

\section{Comparison between LSR and OC-LAR (Fig. 1)}

Individual $\mathrm{GH}$ concentrations decreased to less than $2.5 \mu \mathrm{g} / \mathrm{l}$ in one patient (No. 8) during LSR and in four patients (Nos 6, 8, 10 and 12) during OC-LAR treatment. During both treatments, the variability among GH concentrations in the three samples collected at each time point was very low (data not shown), being $0.5-1 \mu \mathrm{g} / \mathrm{l}$ in most sensitive patients (Nos $1,4,5,6,8$ and 10-12).

LSR treatment achieved normal age-adjusted IGF-I concentrations in four patients (Nos 5, 8, 10 and 11). OC-LAR achieved normal IGF-I concentrations in five patients (Nos 4, 5, 8, 10 and 11), in three of them (Nos 5, 8 and 10) with $10 \mathrm{mg} /$ cycle, and near-normal (i.e. $<110 \%$ ULNR) in three other patients (Nos 1, 2 and $12)$.

\section{Side effects}

No relevant side-effect was recorded during either LSR or OC-LAR treatments. Fasting plasma glucose and $\mathrm{HbA}_{1 \mathrm{c}}$ concentrations did not change during either LSR or OC-LAR treatment (data not shown). At ultrasound 
(a)

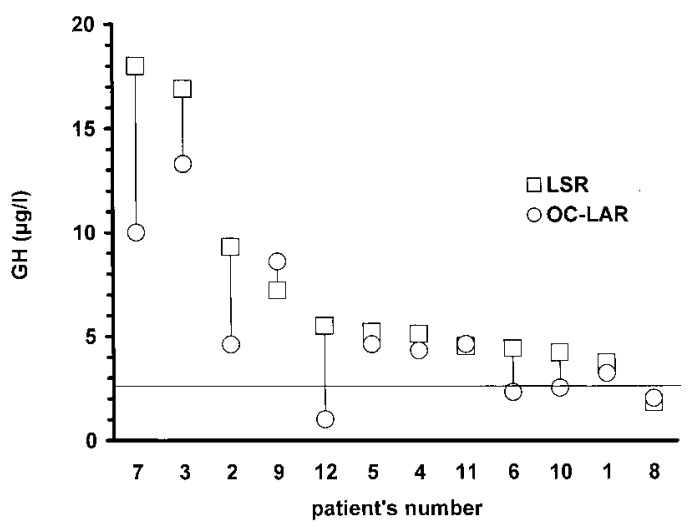

(b)

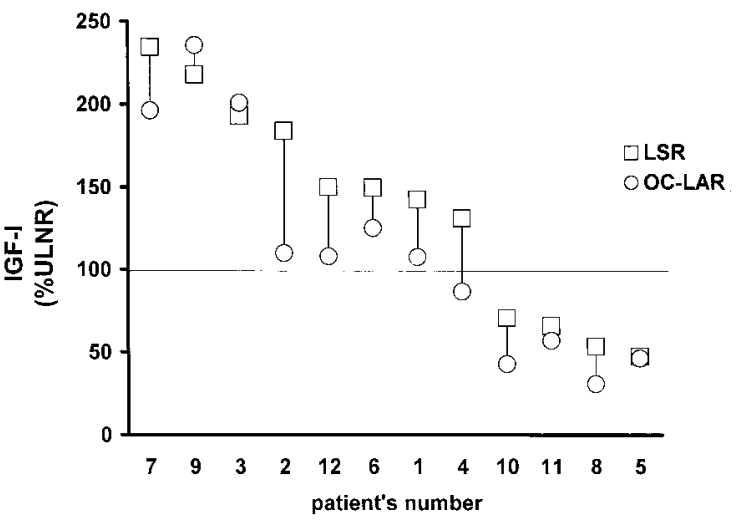

Figure 1 Individual GH concentrations (a) and IGF-I concentrations expressed as \%ULNR $(b)$ during LSR and OC-LAR treatment. $\square$, hormonal value obtained during LSR; $O$, hormonal value achieved during OC-LAR, for each patient. The horizontal line is set at $2.5 \mu \mathrm{g} / \mathrm{l}$ in (a) and at $100 \%$ ULNR in (b).

examination, no gallstone was observed either before or during either somatostatin analog treatments.

\section{Discussion}

Somatostatin analogs have dramatically improved medical treatment in acromegaly. The multiple (twice or three times daily) injection schedule has achieved a reasonable control of acromegaly in most patients, with normalization of $\mathrm{GH}$ and IGF-I concentrations in $30-60 \%$ of treated patients and a reduction in tumor size in $20-50 \%(6,7)$. Continuous s.c. infusion of octreotide controlled the escalation of hormonal concentrations that occurred $6-8 \mathrm{~h}$ after individual injections, obtaining a better control of the disease, often with lower daily doses of the drug, but with the burden of discomfort (8). The development of the long-acting somatostatin analog LSR has allowed stable control of acromegaly by means of injections administered at 1014-day intervals $(3,9)$. Nowadays, OC-LAR is known to be very effective with a once-monthly administration schedule (5). Our study is the first that aimed to compare directly the two available long-acting somatostatin analogs in the same series of acromegalic patients.

Our data show that OC-LAR treatment achieves a tighter control of hormonal concentrations as compared with LSR; indeed, in our series, OC-LAR achieved a greater suppression of $\mathrm{GH}$ and IGF-I values. Even though the difference in mean values between the two drugs might not seem to be relevant, safe GH concentrations $(<2.5 \mu \mathrm{g} / \mathrm{l}(10))$ and normal or near normal $(<110 \%$ ULNR $)$ IGF-I concentrations were indeed reached in a greater proportion of patients (33\% compared with $8 \%$ for $\mathrm{GH}$ and $66 \%$ compared with 33\% for IGF-I) during OC-LAR.

Lanreotide and octreotide exert their effects on $\mathrm{GH}$ secretion by coupling to the same pituitary somatostatin receptors (SST-R) of the subtypes 2 and 5 (11), showing similar affinity and binding properties. Therefore, the finding of unequal GH/IGF-I suppression during these two different treatments points to a more powerful or more prolonged inhibition, or both, of GH secretion by OC-LAR administration and consequently a greater extent of IGF-I normalization.

The results obtained in our patients during LSR treatment are far less satisfactory than those previously reported in other series $(3,9)$. Even though a marked decline of hormonal values was observed, our values for normalized GH and IGF-I concentrations are low, in spite of the shorter interval of drug administration (at 7-10 days in most patients) as compared with the scheduled prescription. This result may reflect the small size of the series and on our having adopted the tighter criteria of cure for acromegaly that have been recommended in the past few years (1) - that is, GH concentrations less than $2.5 \mu \mathrm{g} / \mathrm{l}$ (10), and IGF-I matched for age (12).

The analysis of individual response to the two somatostatin analogs shows heterogeneous responses: some patients (Nos 5, 8 and 10) were very sensitive to somatostatin analogs, achieving normalization of IGF-I values with the 14-21-day schedule during LSR treatment and during $10 \mathrm{mg}$ OC-LAR treatment, whereas others (Nos 3, 7 and 9) showed a lower degree of sensitivity to somatostatin analogs, as pathological GH and IGF-I concentrations persisted even with the highest OC-LAR dosages $(30 \mathrm{mg})$.

The study design (direct switch from LSR to OC-LAR without wash-out) could impose a major criticism on the evaluation of our results. Indeed, in acromegaly it is a well-known phenomenon that chronic treatment with somatostatin analogs induces a progressive increase in GH/IGF-I suppression (13), so that the greater hormonal inhibition during OC-LAR treatment could be partially dependent on this finding, a carryover effect of LSR treatment, or both. However, all patients had undergone a very prolonged (median 12 months) LSR treatment at individually tailored dosages, 
that had attained the maximal GH/IGF-I inhibition, as demonstrated by the lack of any changes in hormonal values between the last two control measurements performed during LSR treatment. Therefore, we believe that the further GH/IGF-I suppression with the short OC-LAR treatment ( 6 months) can be attributed, with confidence, to this drug alone.

An unexpected finding of our study was that, in the patients most sensitive to these new long-acting somatostatin analogs - those in whom $\mathrm{GH}$ values are less than $2.5-3 \mu \mathrm{g} / \mathrm{l}$ and IGF-I are normal or near normal - the assessment of $\mathrm{GH}$ concentrations revealed very stable values. Indeed, the analysis of variability of $\mathrm{GH}$ values among the three samples collected in each patient at each time point of the study procedure showed that, the lower were the hormonal values, the lower was the magnitude of variability. It is still being debated how many samples should be taken for evaluation of the degree of $\mathrm{GH}$ hypersecretion in acromegalic patients, even during medical treatment. In the common clinical setting of outpatients, several samples are usually collected, in the fasting condition and in the first hours of the morning. Even though we did not study the integrated concentrations of GH over a 24-h period (which was beyond the aim of this study), the marked stability of GH concentrations obtained on the three hourly samples during the whole study period in most sensitive patients, together with normal IGF-I values, enable us to hypothesize that long-acting somatostatin analogs flatten the $\mathrm{GH}$ secretory spikes that are typical of acromegaly (14), and to suggest that the follow-up of patients with acromegaly can be simplified, relying on a single morning blood sample that assesses both GH and IGF-I.

In conclusion, these preliminary results obtained in a small series show that OC-LAR is able to produce a control of hormonal hypersecretion in acromegaly that is better than that achieved by LSR in a greater number of patients. This drug is a very promising therapeutic tool that improves the compliance of acromegalic patients inasmuch as it can be administered once every 4 weeks.

\section{References}

1 Melmed S, Jackson I, Kleinberg D \& Klibanski A. Current treatment guidelines for acromegaly. Journal of Clinical Endocrinology and Metabolism 199883 2646-2652.
2 Newman CB, Melmed S, George A, Torigian D, Duhaney M, Snyder P et al. Octreotide as primary treatment for acromegaly. Journal of Clinical Endocrinology and Metabolism 199883 30343040.

3 Giusti M, Gussoni G, Cuttica CM, Giordano G \& the Italian Multicentre Slow Release Lanreotide Study Group. Effectiveness and tolerability of slow release lanreotide treatment in active acromegaly: six-month report on an Italian multicentre study. Journal of Clinical Endocrinology and Metabolism 199681 20892097.

4 Lancranjan J, Bruns C, Grass P, Jacquet P, Jervell J, Kendall-Taylor $\mathrm{P}$ et al. Sandostatin-LAR: a promising therapeutic tool in the management of acromegalic patients. Metabolism 199645 6771 .

5 Flogstad AK, Halse J, Bakke S, Lancranjan J, Marbach P, Bruns C et al. Sandostatin LAR in acromegalic patients: long-term treatment. Journal of Clinical Endocrinology and Metabolism 1997 82 23-31.

6 Ezzat S, Snyder PJ, Young WF, Boyajy LD, Newman C, Klibanski A et al. Octreotide treatment of acromegaly: a randomized multicenter study. Annals of Internal Medicine 1992117 711-718.

7 Newman B, Melmed S, Snyder PJ, Young WF, Boyajy LD, Levy R et al. Safety and efficacy of long-term octreotide therapy of acromegaly: results of a multicenter trial in 103 patients - a clinical research center study. Journal of Clinical Endocrinology and Metabolism $1995802768-2775$.

8 Roelfsema F, Frolich M, de Boer H \& Harris AG. Octreotide treatment in acromegaly: a comparison between pen-treated and pump-treated patients in a cross-over study. Acta Endocrinologica $199112543-48$

9 Caron P, Morange-Ramos I, Cogne M \& Jacquet P. Three year follow-up of acromegalic patients treated with intramuscular slow-release lanreotide. Journal of Clinical Endocrinology and Metabolism 199782 18-22.

10 Bates AS, Van't Hoff W, Jones JM \& Clayton RN. An audit of outcome of treatment in acromegaly. Quarterly Journal of Medicine $199386293-299$.

11 Reisine T \& Bell GI. Molecular biology of somatostatin receptors. Endocrine Reviews $199516427-442$.

12 Barkan AL, Beitins IZ \& Kelch RP. Plasma insulin-like growth factor-I/somatomedin C in acromegaly: correlation with degree of growth hormone hypersecretion. Journal of Clinical Endocrinology and Metabolism 198867 69-73.

13 Lamberts SWJ, Uitterlinden P \& Del Pozo E. SMS 201-995 induces a continuous decline in circulating growth hormone and somatomedin-C levels during therapy of acromegalic patients for over two years. Journal of Clinical Endocrinology and Metabolism $198765703-710$.

14 Ho PJ, Jaffe CA, Demott Friberg R, Chandler WF \& Barkan AL. Persistence of rapid growth hormone pulsatility after successful removal of GH-producing pituitary tumors. Journal of Clinical Endocrinology and Metabolism 199478 1403-1410.

Received 19 April 1999

Accepted 10 June 1999 